\title{
The Researches on Food Traceability System of University takeout
}

\author{
Jia xin $l u^{1, *}$, Ce zhao ${ }^{1}$, Zhuang zhuang $\mathrm{li}^{1}$,Zi rong shao ${ }^{1}, \mathrm{Kun}$ yi $\mathrm{pi}^{1}$ \\ ${ }^{1}$ Shan dong Jiao tong University, Ji nan, Shandong 250300, China
}

\begin{abstract}
In recent years, campus takeaway has developed rapidly, and all kinds of online ordering platforms are running. The problem of distribution in the campus can not only save the time cost of the businessmen, but also guarantee the effective management of the school, which is beneficial to the construction of the standard health system for the takeout. But distribution according to the existing mode will cause certain safety and health risks. The establishment of the University takeaway food traceability system can solve this problem. This paper first analyzes the sharing mode and distribution process of campus takeaway, and then designs the intelligent tracing system for the campus takeaway; the construction of the food distribution information platform and the problem of the recycling of the green environment of the dining box. Finally, the intelligent tracing system of the school takeout is analyzed with the braised chicken as an example.
\end{abstract}

\section{Introduction}

In recent years, the $\mathrm{O} 2 \mathrm{O}$ online ordering platform represented by the US group takes the first place to grab the campus market, and is welcomed by the students. The reasons for the rapid expansion of this new consumption pattern in the campus include three points: first, the rapid development of the information technology, the popularization of electronic commerce, the student groups in Colleges and universities are also easy to accept new things, and more convenient for the promotion of new consumption patterns; second, the phenomenon of regional gathering in universities and colleges is remarkable, and the catering industry has also gathered around it in order to meet the needs of students. Therefore, it is easy to concentrate the distribution of logistics management; third, the students' lifestyle is diverse, busy learning or weather reasons, such as unwilling to go to the dining room, the takeout provides convenience [1]. The delivery of logistics in the campus can not only save the time cost of the businessmen, but also guarantee the effective management of the school, help the construction of the standard health system, and reduce the cost of the students' living and facilitate the life of the students.

\section{Analysis of Campus Takeout Sharing Mode and Distribution Process}

\subsection{Analysis of Campus Takeout Sharing Mode and Distribution Process}

The takeaway E station mode we use is a smart common distribution mode, as shown in Figure 1.

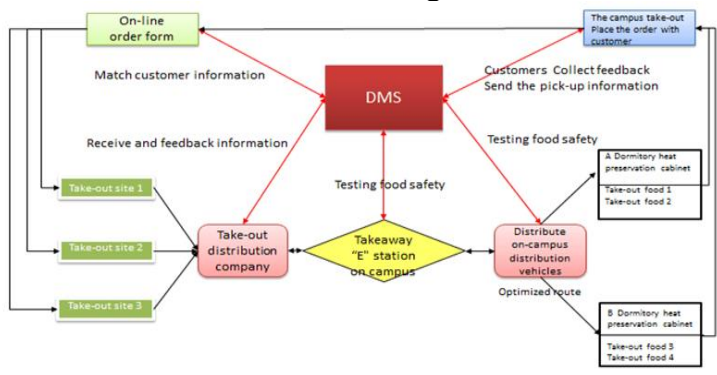

Fig. 1. Campus Takeout Operation Pattern

After the order information is completed and the order information is generated. This order information is sent to the store for takeout and distribution, and then the goods are taken away from the logistics company to the takeout merchant and sent to the E station for the campus takeaway. Through the campus cloud platform management system, the order information is matched with the campus customer information (including address information, telephone, etc.), and then assigned to the school distribution personnel who is to distribute the takeaway to the insulation cabinet of the various school floors. This kind of access cabinet is usually set in the dormitory building, which separates the student's living area from the learning area. In addition, it also separates the problems arising from the arrival of the foreign distribution personnel after the dormitory building. When the takeaway food is distributed to the insulation cabinet, the system automatically identifies the order information and sends SMS prompts to the customers. When the customers take out, they can pick up the goods according to the two-dimensional code or password provided by the SMS, which not only ensures

\footnotetext{
* Corresponding author: 971362610@e-mail.org
} 
the punctuality, safety, optimization of the road, but also creates a good takeout environment for the students in the school. To automate the process of distribution and sub inspection, so as to improve the efficiency and reduce the artificial link, and the staff will concentrate on a work point, which can improve the efficiency of work.

\subsection{Analysis of the operation mode of E station in campus takeout}

Analysis on the operation mode of the campus takeaway e station as shown in Figure 2.

Try to ensure that lines are no thinner than 0.25 point.

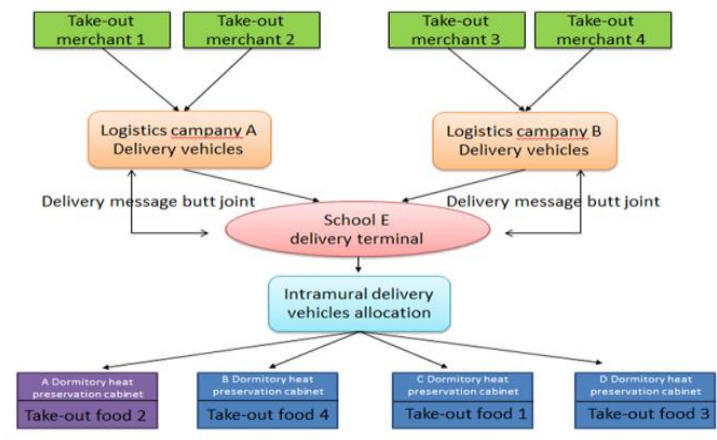

Fig. 2. "One-to-many" recycling distribution pattern

The distribution information of the day is displayed on the GIS electronic map by GIS, and then the position of student dormitories, the number of orders and the location of the order are analyzed with the GIS spatial analysis function. Finally, the location of the distribution center, the traffic situation of the road, the number of vehicles and so on are analyzed, and the genetic algorithm is used to advance the distribution route.

After the delivery route is determined, the number of customers on each route, the number of orders, the total volume of distribution goods and so on will also be determined. According to these parameters and the status of vehicles and personnel in the distribution center, the loading plan can be decided.

\section{Design of intelling traceability system for campus takeout}

\subsection{System concept design}

As the whole takeout begins, the visualization and traceability of information must be embodied, so that we can reasonably control the problems of each link and trace the whole process. When a problem occurs in a process, stop distribution in time, so as to minimize the crisis and improve service quality. At present, many takeaway systems are complex, and food safety is not guaranteed. After the food crisis is found, the users do not know where the source is, and the responsible person of each link has unloaded the responsibility, which leads to the weakness of the awareness of rights, the lack of means of safeguarding rights and the higher cost of safeguarding rights. Most customers take a tolerant attitude, and just few people really pursue it, so a complete traceability system is needed for the current takeaway system. Figure 3 is the system concept design, which is divided into one system and seven processes.

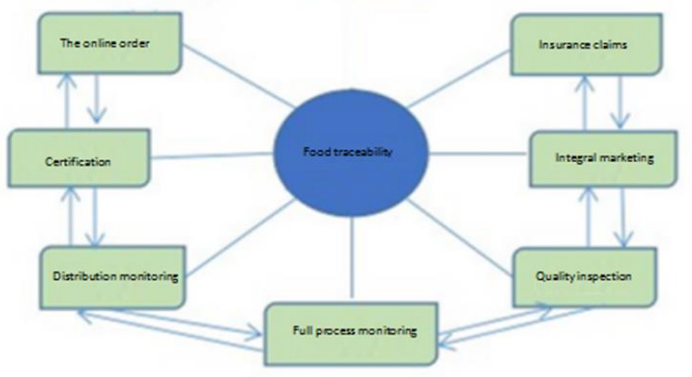

Fig. 3. Campus Takeout Operation Pattern

First, a traceability system is built based on cloud platform data, which is divided into seven parts based on online monitoring. The emphasis is on the traceability of the intramural takeout, encoding from the takeaway merchants to the completion of the distribution. If the potential crisis is found, the distribution is terminated immediately, and the safety monitoring of the material, the inspection of the distribution link and the inspection of the service process are carried out. Besides, it can also score marketing. If the takeaway business successfully completes an order, its score will rise. The higher the score is, the higher the credit value of the takeaway business will be, and then the merchant will be promoted to the app home page, so that more people can buy at ease. If the food safety problem appears in the link, it can be traced back to every link of distribution, and can reduce the loss according to the insurance claim, realize the minimization of the impact, and control the problem of distribution. The whole distribution and the traceability process are shown in Figure 4.

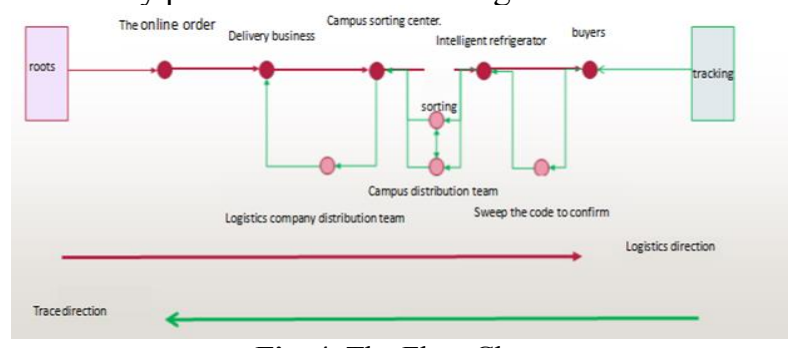

Fig. 4. The Flow Chart

\subsection{Two dimensional code tracing process}

Product traceability is to record its history, and the location should be querying. The recording system is carried out well and executed well, and the traceability of products is also realized. First, load information, combine the feature encryption logo with the product, and give the unique barcode and encrypted digital information of each takeaway by software. Next, we use bar code as a carrier to input and upload every link information to the traceability system of product quality information. Before the transport, sorting, and distribution links, ensure that each link has generated data and uploaded, and then supervised by the specialized personnel. In the final arrival of the intelligent takeaway cabinet, the takeaway generates a bar code containing all the links. Finally, the whole 
process can be traced by scanning code, and consumers can scan the whole process of takeaway food distribution by scanning WeChat.

Through two dimensional code, the information of each circulation node can be interconnected to form a chain of quality safety traceability, and "point supervision" is transformed into "chain supervision" "face supervision", which will be more conducive to the quality supervision of product quality and improve the quality of products.

\subsection{Safety traceability guarantee the rights and interests of consumers}

The quality safety traceability system of takeaway food is an information management system that can connect production, inspection, supervision and consumption, so that consumers can understand the process of regular production and circulation, and improve the degree of consumers' reassurance. The system provides the traceability model of "from distribution to table", extracts the elements of supply chain links such as production, processing, circulation and consumption, and establishes a food safety information database. Once problems are identified, effective control and recall can be traced back to source, so as to protect consumers' legitimate rights and interests from the source.

(1)The advantage of two-dimensional code is used to achieve the management of food safety and traceability, so that it is more efficient, real-time and convenient.

(2) Using the two dimensional is to provide completely transparent management ability in food supply chain, to ensure the whole process of food safety visual control, and to call back the problem food.

(3)It can provide basis for effective assessment and scientific early warning of potential potential food safety hazards.

(4)The data can be reported in real time and accurately through the network, so it is convenient to do deeper analysis and research quickly and efficiently.

(5)Through the Internet, consumers can query the complete tracking information of the food purchased.

\section{Taking the braised chicken as an example to analyse the intelligent tracing of the campus takeout}

The data show that the "braised chicken set meal" of the takeout food of Shandong Jiaotong University is the most, and because of various factors that lead to the safety problems such as decay and deterioration, we use the "braised chicken set meal" as an example to reflect the intelligent tracing of the scheme. The basic process is shown in Figure 5.

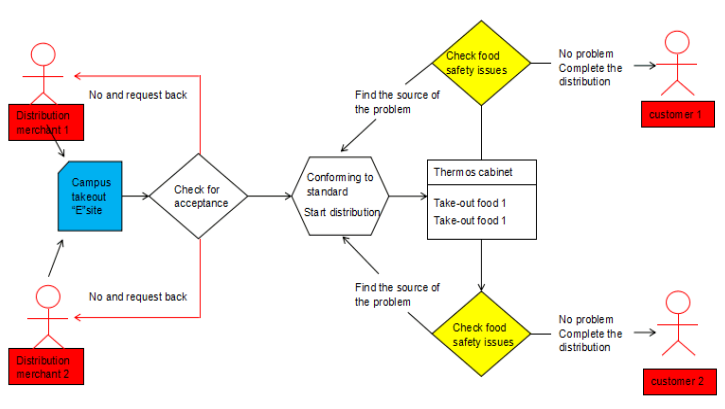

Fig. 5. Retrospective flow chart

The safety problems of "yellow braised chicken set meal" can be generally divided into food safety, safety of production process, packaging safety and distribution freshness. Food safety can only be controlled by takeaway businesses, which is only suitable for food distribution in campus. If this link detects the security problem, then go back to the last step. So this plan is tracking the freshness of distribution, packaging safety and food safety.

The scheme uses two-dimensional code to complete the collection of tracking information. The takeaway from outside school enters the sorting center of the school. We scan two-dimensional code to obtain food information and detect food safety issues, such as food that meets the provisions of the national food safety law and the business license of the business. The order that will affect the safety of takeaway will be returned in time, and the "yellow braised chicken set" that meets the food safety regulations will enter the school delivery section. When sorting points confirm that "yellow braised chicken set meal" is safe, we start distributing, updating APP's information and two-dimensional code information. If there are problems in the delivery process, the reason for the problem should be in the school delivery process, and the loss is borne by the company responsible for the school distribution. Another important safety problem is that's major safety problems such as discomfort or food poisoning after eating "yellow braised chicken set meal". Tracking system should be traced throughout the whole process to find the source of food safety problems. We will use relevant laws to safeguard students' rights, and solve the problem of takeaway food safety through this plan.

The common distribution mode of campus disrupts the principle of separate distribution by the takeaway company, and adopts the unified sorting according to the criteria of regional distribution, and generates twodimensional code of user related information and is attached to the package of the takeaway. Sorting is done through the sorting device according to the takeaway place. Arrange vehicles to distribute the cabinets reasonably. The cabinets can identify the user's personal information by two-dimensional code and send messages automatically to inform the students to take out, or send a meal to the door, and to identify whether the type of takeaway needs to be cooled or heated and treated accordingly. Students can get their takeaway food only by certification and recognition. It can also recycle the lunch box, make use of environmental protection, and 
solve the pollution problem of a large number of lunch boxes.

\section{Conclusion}

The project always adheres to the development concept of "green, safe, low carbon and efficient". On the basis of the existing research results, the project will continue to pay attention to the need of the campus and the society for the delivery mode and the "last mile" distribution problem. The project studies the key technical equipment and application problems, and endeavours to build the project into a demonstration project of the integrated and interactive operation of the teaching, scientific research and social services, and to guide the development of the distribution of the campus takeout to the standard and safe direction. Through the demonstration and popularization and application of the project, we will build our domestic influential campus takeaway brand. After that, the project is popularized and designed according to the specific circumstances of other campus. Finally, all the campus modes will be integrated and the operation mode of the large ring is set up.

\section{References}

1. Liu Junhua, Wang Yanfeng, du Juan, Tian Zhihong.Research on Food traceability system based on Information sharing.2006.12.16.

2. Feng Xueyuan, Zhang Rujia, Gu Yuefang.Investigation and improvement suggestion on campus express logistics of comprehensive university [J].Hong Kong and Macao economy.2014 11: 63-64.

3. Li Xiaojuan.Study on the implementation of Green Logistics [J].Shandong textile economy.2017,(1):5-6.

4. Lei Yun, Tu Qinghua, Song Junfei, Zhong Yuan.Construction and Research of Intelligent Campus Service platform in big data era [J].Communications World. 2017: 275-276.

5. Huo Wen-jin.Research on Logistics Distribution Management system based on RFID.2010. 